\title{
A Behavioral Role for Feature Detection by Sensory Bursts
}

\author{
Gary Marsat and Gerald S. Pollack \\ Department of Biology, McGill University, Montreal, Quebec, Canada H3A 1B1
}

\begin{abstract}
Brief episodes of high-frequency firing of sensory neurons, or bursts, occur in many systems, including mammalian auditory and visual systems, and are believed to signal the occurrence of particularly important stimulus features, i.e., to function as feature detectors. However, the behavioral relevance of sensory bursts has not been established in any system. Here, we show that bursts in an identified auditory interneuron of crickets reliably signal salient stimulus features and reliably predict behavioral responses. Our results thus demonstrate the close link between sensory bursts and behavior.
\end{abstract}

Key words: sensory coding; neuroethology; cricket; behavior; auditory; predator detection

\section{Introduction}

Bursts occur in a number of sensory systems (Eggermount and Smith, 1996; Yu and Margoliash, 1996; Sherman, 2001; Martinez-Conde et al., 2002; Chacron et al., 2004; Krahe and Gabbiani, 2004), and it has been proposed that they constitute a specific neural code, signaling the occurrence of behaviorally relevant sensory events. The possible coding functions of sensory bursts have been studied particularly thoroughly in the lateral geniculate nucleus (LGN) of mammals and in the electrosensory lateral line lobe (ELL) of weakly electric fish. Bursts by relay cells of the LGN signal large changes in light intensity, typical of natural moving scenes (Lesica and Stanley, 2004). In pyramidal cells of the ELL, bursts preferentially encode slow modulations in the amplitude of the fish's electric field, similar to the field distortions that are caused by prey (Oswald et al., 2004). In these examples, bursts extract and signal features of the stimulus that are thought to be behaviorally important (Guido et al., 1995; Lesica and Stanley, 2004; Oswald et al., 2004), but the link between bursting and behavior remains elusive. Indeed, a behavioral role of sensory bursts has not yet been established for any system.

Echolocating bats hunt flying insects using ultrasonic probes and crickets and, like many nocturnally flying insects, respond to ultrasound stimuli with avoidance responses (Hoy, 1992), a behavior that is triggered by an identified ultrasound-sensitive interneuron, AN2. AN2 occurs as a bilateral pair. Each AN2 receives excitatory input from one ear in the prothoracic ganglion. Its axon ascends ipsilaterally to the brain, in which descending motor commands originate (Brodfuehrer and Hoy, 1989). Previous work showed that high-frequency firing of AN2, which occurs at the onset of an ultrasound stimulus, is required for behavioral responses (Nolen and Hoy, 1984). However, bat-insect

Received May 25, 2006; revised Aug. 17, 2006; accepted Sept. 7, 2006.

This work was supported by the Canadian Institutes for Health Research and the Natural Science and Engineering Research Council of Canada. We thank Drs. M. Chacron, R. Chase, and R. Krahe for their suggestions on a previous version of this manuscript.

Correspondence should be addressed to Gerald Pollack, Department of Biology, McGill University, 1205 Doctor Penfield Avenue, Montreal, Quebec, Canada H3A 1B1. E-mail: gerald.pollack@mcgill.ca.

DOI:10.1523/JNEUROSCI.2221-06.2006

Copyright $\odot 2006$ Society for Neuroscience $\quad$ 0270-6474/06/2610542-06\$15.00/0 encounters may last for several seconds, during which the insect is exposed to continuous, dynamic, stimulation with ultrasound (Simmons, 2005); thus, the need for AN2 to detect behaviorally relevant features of ultrasound stimuli and trigger avoidance responses persists at least for several seconds after the onset of a dynamic stimulus. The response of AN2 to long-lasting stimuli includes both isolated action potentials and bursts. Using signaldetection theory, we show that bursts, but not isolated spikes, detect salient amplitude increases with high accuracy. Moreover, only bursts reliably signal the location of the stimulus, which must be coded to elicit properly directed behavioral responses. Most importantly, we show that bursts, but not isolated spikes, reliably predict behavioral responses.

\section{Materials and Methods}

Behavioral recordings. Teleogryllus oceanicus females, aged 7-14 d after the final molt, were anesthetized by chilling on ice; wings, hindlegs, and midlegs were removed to facilitate behavioral and neurophysiological recordings. Crickets were tethered dorsally and placed ventral-side up in a wind stream to elicit flight behavior, which can be identified, despite the absence of wings, by beating of the wing stumps and by the characteristic flight posture assumed by the antennae and front legs. Abdominal steering movements associated with ultrasound avoidance were monitored by casting a shadow of the abdomen on to a photocell array, the voltage output of which varied approximately linearly with lateral displacement of the abdomen (mean \pm SD slope, $11.8 \pm 1.3 \mathrm{mV} / \mathrm{mm} ; n=3 ; R^{2}=$ 0.85 ). This measurement quantifies the sign and strength of the motor output, but, because the precise relationship between abdominal displacement and flight path is unknown, it does not describe the angle toward which the cricket attempts to turn. Signals were digitized for off-line analysis (16 bits; $10 \mathrm{kHz}$ sampling rate; PCI-6251 analog-todigital board; National Instruments, Austin, TX).

Electrophysiology. Twenty to $40 \mathrm{~min}$ after the behavioral assay, the cricket was prepared for in vivo electrophysiology as described previously (Marsat and Pollack, 2004). AN2 was recorded extracellularly, and bilaterally, from the cervical connectives with stainless-steel hook electrodes. Recordings were amplified with a Grass P15 amplifier (Astro-Med, West Warwick, RI) and digitized as described above. The action potentials of AN2 were easily recognized by their large amplitude and lowest threshold to ultrasound.

Sound stimulation. Sound was broadcast from either of two loudspeakers situated to the right and left of the cricket, perpendicular to the long 
axis. Stimuli (15 s duration) had carrier frequency of $30 \mathrm{kHz}$ and Gaussian amplitude envelopes (low-pass filtered at $200 \mathrm{~Hz}$; SD of $6 \mathrm{~dB}$, except when otherwise noted). Stimuli were generated digitally at sampling rate of $120 \mathrm{kHz}$. Except where specified otherwise, mean stimulus level was 85 $\mathrm{dB}$ sound pressure level (SPL), corresponding to $\sim 20-25 \mathrm{~dB}$ above threshold. Stimuli were preceded by at least $1 \mathrm{~min}$ of silence.

Spike train separation. All analyses are for responses to axon-ipsilateral stimuli, except for those in Figure 3, in which ipsilateral and contralateral responses are compared. We excluded from our analyses the first second of responses (both behavioral and physiological), during which firing rate of AN2 and behavioral responses adapt. Spike trains, expressed as series of ones (for time points at which spikes occur) and zeros, were downsampled to $2 \mathrm{kHz}$ and separated into two new series, consisting either of only isolated spikes (i.e., the points at which spikes within bursts occurred were set to 0 ) or of only spikes within bursts [interspike interval (ISI), $\leq 6.5 \mathrm{~ms}$ ]. For the analyses presented in all figures except Figure 3, $a$ and $b$, only the first spike in each burst was retained, thereby reflecting the timing of the bursts but not their internal structure.

Feature detection. We performed standard feature-detection analysis as described in detail previously (Metzner et al., 1998). We describe the procedure here only for the burst-containing train; the identical procedure was followed for the isolated-spike train. A stimulus vector, $s$, was defined for each successive time point in the burst train as the amplitude envelope of the 150-ms-long stimulus segment preceding that point. Each $s$ was assigned to one of two ensembles according to whether or not, at the reference point, a burst occurred. The means of these ensembles correspond to the burst-triggered (BTA) and no-burst-triggered (NTA) averages. The difference between these ensembles was assessed using a Euclidian classifier. The feature vector, $f$, was defined as BTA - NTA. We projected $s$ on to $f$ to quantify their similarity, yielding the conditional probability distributions $P(f \cdot s \mid$ burst $)$ and $P(f \cdot s \mid$ no burst $)$. Receiver operating characteristic curves were generated by varying a threshold similarity level, $T$. For each threshold value, the probability of detection $\left(P_{\mathrm{D}}\right)$ is calculated as the sum of $P(f \cdot s>T \mid$ burst $)$, and the probability of failure $\left(P_{\mathrm{F}}\right)$ as the sum of $P(f \cdot s>T \mid$ no burst $)$. The error level for each threshold value is $1 / 2 P_{\mathrm{F}}+1 / 2\left(1-P_{\mathrm{D}}\right)$. We report the minimum error level across values of $T$.

Behavior analysis. Recordings of abdomen movements were high-pass filtered with a cutoff frequency of $0.5 \mathrm{~Hz}$ to eliminate slow drifts in abdomen position (Pollack and Plourde, 1982) and focus on the rapid abdomen flexions associated with the avoidance steering response during flight. The behavioral responses were either averaged across repetitions of the same stimulus to calculate spike- and burst-triggered averages or quantified for individual abdominal movement recordings. We quantified behavior in two ways. First, we used the SD of the abdominalmovement recording as a measure of the response. This reflects the strength and frequency of abdomen deflections, including both "correct" responses, i.e., away from the sound source, and misdirected responses. Second, to examine separately correct and misdirected responses, we measured the area under the half-rectified recording of abdomen position separately for deflections away from and toward the sound source. The method based on SD and that based on half-rectified recordings that retained only responses directed away from the source yielded nearly identical results, suggesting that responses were directed mainly away from the sound source. This is confirmed by comparison of half-rectified responses directed toward and away from the sound source (supplemental Fig. 2, available at www.jneurosci.org as supplemental material). Only the results based on SD are presented here; those based on rectified recordings are available in the supplemental data (available at www.jneurosci.org as supplemental material).

Statistical treatment. Response measures (both electrophysiological and behavioral) of individual crickets to a given stimulus were averaged. Averages of these values across crickets are reported in Results and were used for statistical analyses, except for Figure $5 b$. Proportions were arcsine square root transformed (Zar, 1999) before being subjected to statistical tests.
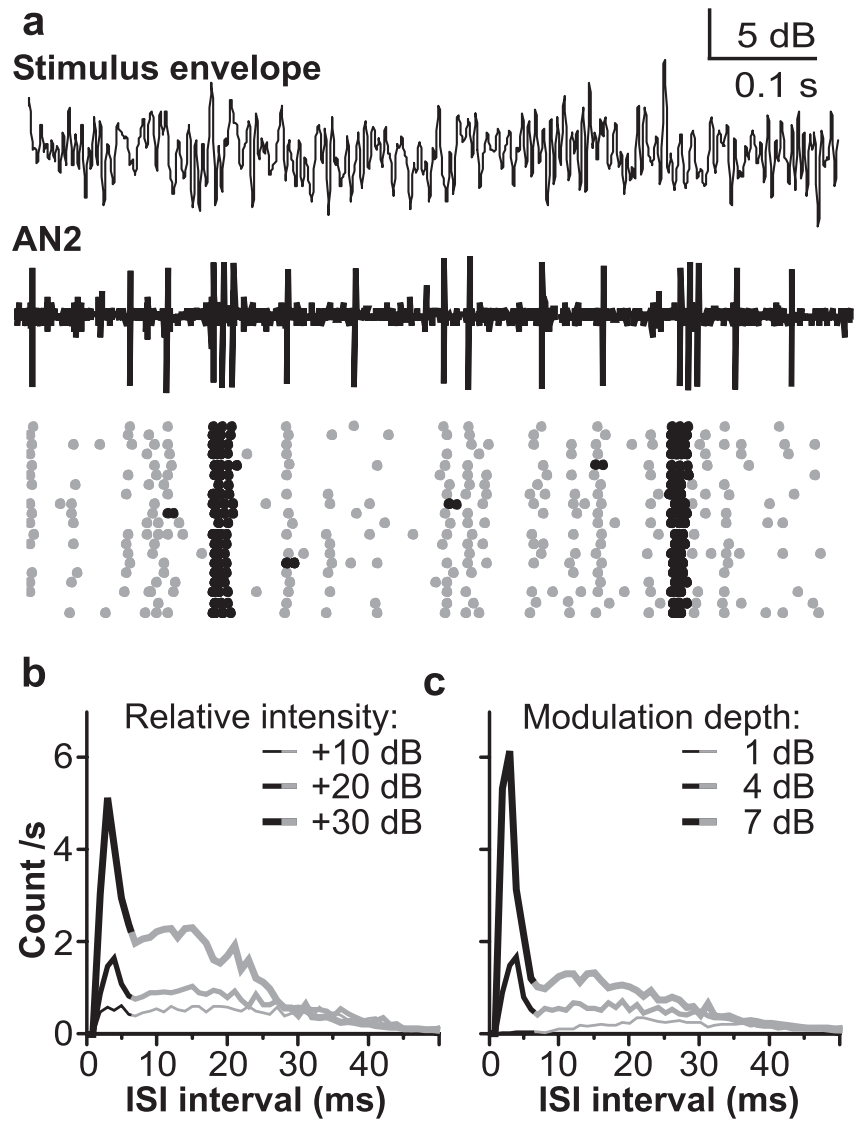

Figure 1. Bursts in AN2 in response to amplitude modulated stimuli. $\boldsymbol{a}$, Excerpt of the response of AN2 to a Gaussian-envelope stimulus, beginning $4 \mathrm{~s}$ after stimulus onset. The top trace shows the amplitude envelope of the stimulus (mean sound level, $85 \mathrm{~dB}$ SPL; SD of $6 \mathrm{~dB}$ ). The middle trace is the response of AN2. The bottom plot shows responses to 20 repetitions of the same stimulus. Gray dots represent isolated spikes; black dots indicate spikes within bursts. $\boldsymbol{b}$, ISI distributions of responses to stimuli that differ in mean sound level (SD of amplitude envelope, $5 \mathrm{~dB} ; n=9$ ). Mean \pm SD firing rates are as follows: $21.9 \pm 2.3,32.7 \pm 2.7$, and $61.7 \pm 4.6 \mathrm{~Hz}$ for stimuli 10,20, and $30 \mathrm{~dB}$ above threshold, respectively. C, ISI distributions of responses to random amplitude modulation stimuli with different modulation depths (stimulus intensity, $85 \mathrm{~dB} \mathrm{SPL} ; n=6$ ). In $\boldsymbol{b}$ and c, ordinate shows number of ISIs per second; bin width, 1 ms. The black portions of the curves represent ISIs of spikes within bursts (ISI of $\leq 6.5 \mathrm{~ms}$ ). Mean \pm SD firing rates are as follows: $12.2 \pm 3.1,26.2 \pm 3.8$, and $48.7 \pm 5.7 \mathrm{~Hz}$ for stimuli with amplitude envelope $S D$ of 1,4 , and $7 \mathrm{~dB}$, respectively.

\section{Results}

Responses to long-lasting stimuli with Gaussian amplitude envelopes consist, after a rapid adaptation phase (Samson and Pollack, 2002), of both isolated spikes, separated by relatively long and variable ISIs, and of bursts, consisting of groups of spikes separated by short ISIs (typically 3-4 ms) (Fig. 1a). Bursting is reflected in ISI histograms (Fig. 1b) as a narrow peak of short ISIs that is distinct from the broader peak of longer ISIs. The proportion of spikes within bursts (operationally defined as spikes occurring with ISI of $\leq 6.5 \mathrm{~ms}$ ) increases with sound level (from $12 \pm 1.3 \%$ for $10 \mathrm{~dB}$ above threshold to $29 \pm 4.1 \%$ for $30 \mathrm{~dB}$ above threshold; means \pm SD; ANOVA, $p=0.012 ; n=9$ ) (Fig. $1 b$ ). Bursting also increases with modulation depth (from $1 \pm$ $0.2 \%$ for envelope SD of $1 \mathrm{~dB}$ to $37 \pm 5.2 \%$ for SD of $7 \mathrm{~dB}$; ANOVA, $p=0.001 ; n=6$ ) (Fig. $1 c$ ).

\section{Bursts function as feature detectors}

We first asked whether, as in other systems, bursts in AN2 might function as feature detectors. Spike-triggered and burst-triggered 

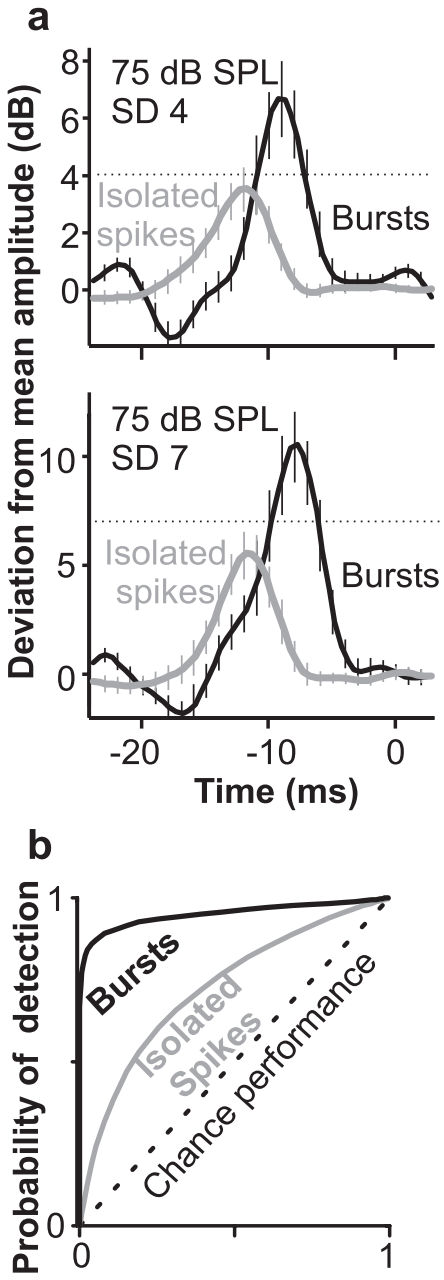

Probability of failure

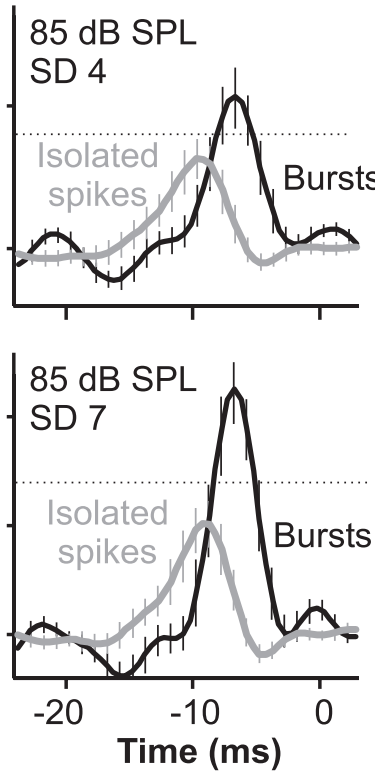

C

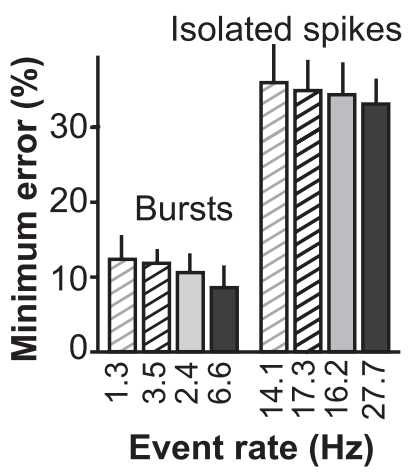

Figure 2. Coding of stimulus features by bursts and isolated spikes. $\boldsymbol{a}$, Spike-triggered averages of the stimulus envelope preceding isolated spikes (gray) or bursts (black) for stimuli with different mean sound levels and modulation depths (mean \pm SD; $n=6$ neurons). Modulation depth is quantified as the SD of the stimulus and is indicated on the plots by dotted lines. Values represent departures from mean stimulus level (indicated on each panel). $\boldsymbol{b}$, Receiver operating characteristic curves, which plot the probability of correct detection of a feature (i.e., a featurecontaining stimulus segment is followed by the appropriate event) against the probability of failure (absence of event after the putative feature), as the stringency for feature identification is varied. The dotted line indicates chance feature detection; performance increases with the degree of departure from this. Stimulus, $85 \mathrm{db}$ SPL; envelope SD, $6 \mathrm{~dB}$ (mean of 16 neurons). $c$, Minimum error level (mean $\pm S D ; n=6$ neurons) for stimuli with different mean intensities (gray, $75 \mathrm{~dB}$ SPL; black, $85 \mathrm{~dB}$ SPL) or modulation depths (hatched bars: envelope SD of $4 \mathrm{~dB}$; filled bars, envelope SD of $7 \mathrm{~dB}$ ). Burst rate or isolated-spike rate is indicated for each stimulus.

averages of prespike or preburst segments of the stimulus envelope show that both bursts and isolated spikes are time locked to increases in stimulus amplitude (Fig. 2a). However, bursts occur after large increases in stimulus amplitude, whereas isolated spikes follow smaller peaks. The amplitude increase required to elicit bursts scales with mean stimulus amplitude and modulation depth such that, for a variety of stimuli, bursts occur after conspicuous amplitude increases, as indicated by peak preburst amplitudes that extend at least 1 SD beyond the mean. Moreover, the peak of the burst-triggered average, but not of the spiketriggered average, is preceded by a period of low stimulus amplitude, as indicated by the dip below the mean level $\sim 15 \mathrm{~ms}$ before the burst.

We used signal-detection measures (Metzner et al., 1998) to

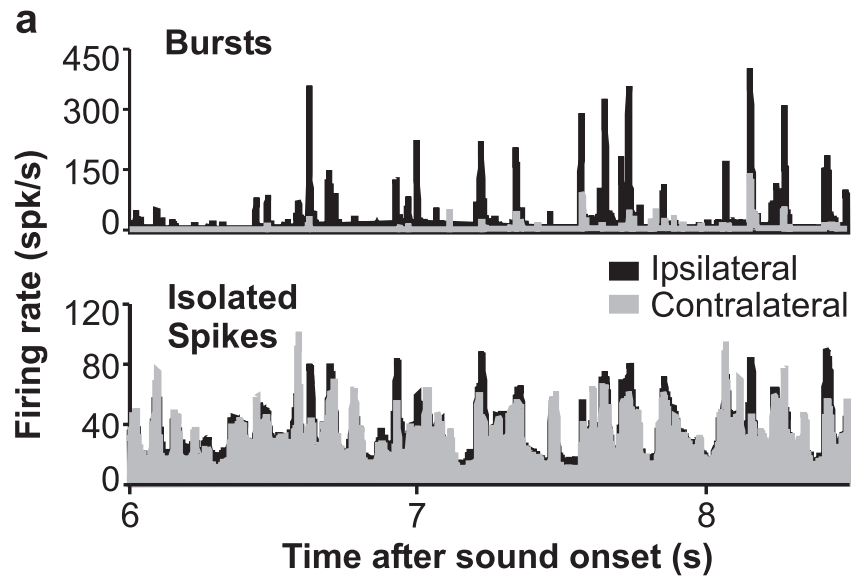

b

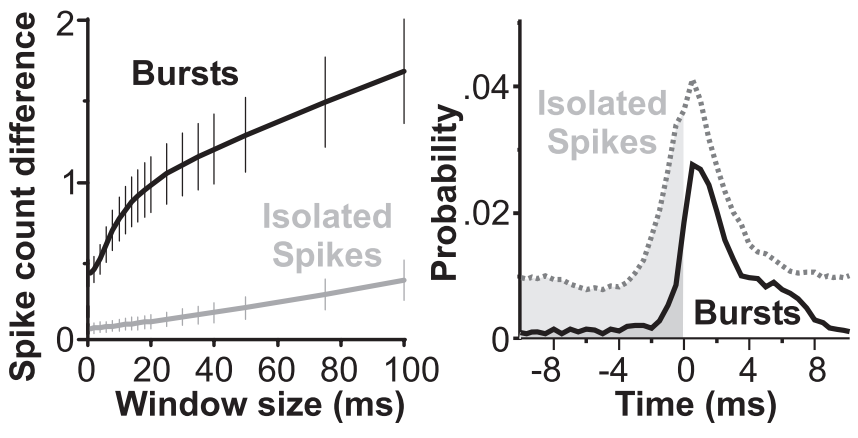

Figure 3. Directionality of the response of AN2. $\boldsymbol{a}$, Mean instantaneous firing rates (calculated as $|S|^{-1}$; example from one experiment, $n=20$ stimulus repetitions) based on either spikes within bursts (top) or isolated spikes (bottom). Both AN2s were recorded simultaneously. $\boldsymbol{b}$, Bilateral spike count difference (mean $\pm S D ; n=16$ neurons) based on isolated spikes or bursts, calculated for sliding windows of different widths. c, Probability of occurrence of isolated spikes (dashed line) or bursts (solid line) in the sound-contralateral AN2 relative to the timing of an isolated spike/burst in the ipsilateral AN2 (bin width, $0.5 \mathrm{~ms}$ ). Shading indicates contralateral-leading events.

quantify the performance of bursts and isolated spikes as feature detectors. Receiver operating characteristic curves (Fig. 2b) show the reliability with which portions of the stimulus resembling features that are putatively associated with bursts or with isolated spikes are in fact followed by these responses. Bursts perform well as feature detectors, whereas isolated spikes do so poorly. Minimum error rate, which includes both failure to identify a feature when it occurs and erroneous identification of a feature when there is none, is $9 \pm 1.6 \%$ for bursts and $33 \pm 3.1 \%$ for isolated spikes for the dataset shown in Figure $2 b$ (means \pm SD; paired $t$ test, $p<10^{-5} ; n=16$ neurons). Although burst rate varies with mean stimulus amplitude and modulation depth (Fig. 1b,c), the accuracy of feature detection by bursts and isolated spikes remains constant over a wide range of stimulus statistics (ANOVA on minimum error level, $p=0.23$ and $p=0.34$ for bursts and isolated spikes, respectively; $n=6$ neurons) (Fig. $2 c$ ).

\section{Bursts encode localization cues}

Ultrasound-avoidance responses are directed away from the sound source. Thus, if bursts are instrumental in driving behavior, they should not only detect a stimulus but also code for its location. Localization of ultrasound is based on bilateral differences in the responses of the left and right AN2s (Pollack, 1998). We quantified localization cues by recording simultaneously from both neurons. Figure $3 a$ shows firing rates of the two neu- 
rons, calculated separately for isolated spikes and for spikes within bursts. The bilateral difference is striking for bursts but is less evident for isolated spikes. Using sliding windows [1-100 ms wide, a range that includes behavioral latencies (Nolen and Hoy, 1986) and thus the integration times of downstream circuits], we calculated separately, for bursts and isolated spikes, the binaural difference in mean spike count per window (excluding windows that contained no spikes on either side). Bilateral spike-count difference is greater for bursts than for isolated spikes over the entire range of window widths (ANOVA, $p=0.001$; Tukey's test, $p<0.05$ for all points) (Fig. $3 b$ ); indeed, the difference for isolated spikes does not differ significantly from zero for any window width (one-sample $t$ test, $p>0.05$ ). Another possible soundlocalization cue is the bilateral timing difference. Figure $3 c$ shows the temporal distribution of spikes or bursts in the AN2 contralateral to the sound source relative to those in its ipsilateral counterpart. We focus on bilateral differences within the range of $\pm 10 \mathrm{~ms}$, which encompasses the range of previously measured binaural latency differences of AN2 for trains of discrete sound pulses (Samson and Pollack, 2002). For 23\% of ipsilateral bursts, a contralateral burst occurs within $\pm 10 \mathrm{~ms}$ (the area under the curve). The sound-ipsilateral burst leads in $85 \pm 5.1 \%$ of these cases (unshaded area) (significantly different from chance, onesample $t$ test, $p<10^{-4}$ ). Thirty-eight percent of ipsilateral isolated spikes are matched by contralateral isolated spikes, but the ipsilateral spike leads in only $61 \pm 8.3 \%$ of these cases, which does not differ significantly from that expected by chance $(p=0.24)$. Thus, based on both spike count and timing, only bursts provide reliable cues for sound direction.

\section{Bursts predict behavior}

To study the role of bursts in behavior, we compared neural and behavioral responses to dynamic stimuli. We recorded a conspicuous component of the ultrasound avoidance response, abdominal movements away from the sound source during tethered flight (Moiseff et al., 1978). Subsequently, we recorded, in the same cricket, the response of AN2 to the same stimulus that was presented during behavioral tests. Visual comparison of behavioral and neural responses suggests that conspicuous abdominal movements tend to follow stimulus segments that elicit bursts in AN2 (Fig. $4 a$, highlighted in black). This is confirmed by the burst-triggered average of abdominal position (Fig. $4 b$ ), which shows that a large abdominal flexion begins $\sim 40 \mathrm{~ms}$ after a burst. This value is similar to that reported by Nolen and Hoy (1984) for the latency between the first AN2 spike of a response and activation of abdominal steering muscles $(49 \pm 16 \mathrm{~ms})$. Abdominal movements after isolated spikes are much less pronounced.

To examine the relationship between spike-train structure and behavior more directly, we manipulated the frequency of occurrence of bursts and isolated spikes. The relative frequency of bursts increases both with mean stimulus amplitude and with modulation depth (Fig. $1 c)$. We chose stimuli $(n=8)$ that differed in mean amplitude and/or in modulation depth, such that both burst rate and isolated-spike rate varied across responses to these stimuli but were not correlated with each other $\left(R^{2}=0.03\right.$; $p=0.24$ ) (supplemental Fig. $1 a$, available at www.jneurosci.org as supplemental material). We quantified the behavioral response as the SD of the abdominal-movement recording (Fig. $5 a)$. Behavior varies strongly as a function of burst rate $\left(R^{2}=\right.$ $0.77 ; p<0.10^{-5}$ ) (Fig. $5 b$ ) but is independent of the isolatedspike rate $\left(R^{2}=0.016 ; p=0.4\right)$ (supplemental Figs. $1 b, c, 2$, available at www.jneurosci.org as supplemental material). These a
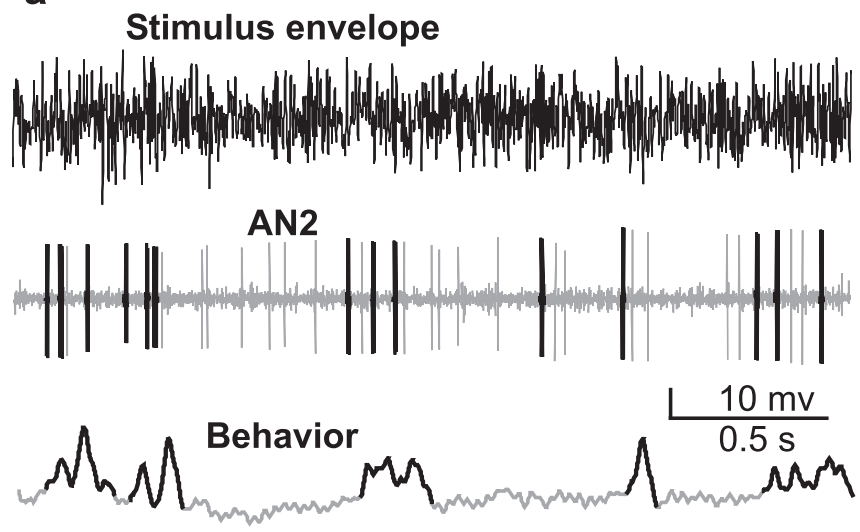

b

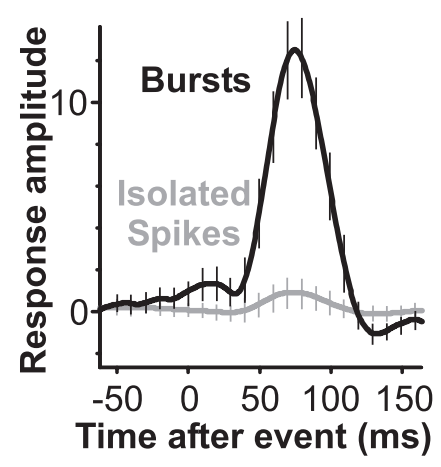

Figure 4. Behavioral impact of bursts and isolated spikes of AN2. $\boldsymbol{a}$, Excerpts of behavioral and neural responses, each beginning $4 \mathrm{~s}$ after onset of the identical Gaussian-envelope stimulus. Note that the neural responses were recorded after completion of the behavioral measurements. Top, Stimulus amplitude envelope; middle, response of the sound-ipsilateral AN2; bottom, abdominal movement. Vertical scale indicates voltage output of the recoding device (see Materials and Methods). Bursts in AN2, and conspicuous abdominal movements away from the sound source, are highlighted in black. $\boldsymbol{b}$, Amplitude of abdominal movement (millivolts of output of recording device; mean $\pm S D ; n=16$ experiments) after an isolated spike (gray) or a burst (black) in AN2. Positive values indicate abdomen flexion away from the sound source.

results argue strongly that AN2 bursts, but not isolated spikes, are responsible for the behavioral responses.

\section{Discussion}

We have shown that bursts in AN2 signal the occurrence of salient peaks in stimulus amplitude, encode sound direction, and predict behavioral responses, all with high reliability. These findings provide strong evidence that bursts are functionally important.

Bursting occurs over a range of stimulus amplitudes and modulation depths, but, in all cases, bursts are triggered by the most conspicuous increases in amplitude. Thus, the stimulus feature that triggers bursts scales with stimulus statistics. Moreover, although the frequency of bursting varies across stimuli, the high accuracy of feature detection remains constant. This stimulusdependent change in the response properties of AN2 is functionally similar to the adaptation that has been described in a number of sensory systems (Smirnakis et al., 1997; Kvale and Schreiner, 2004; Dean et al., 2005), which allows accurate detection and encoding of changes in a stimulus over a wide range of stimulus statistics.

In addition to detecting large amplitude increases, bursts reliably signal the location of the sound source through bilateral 

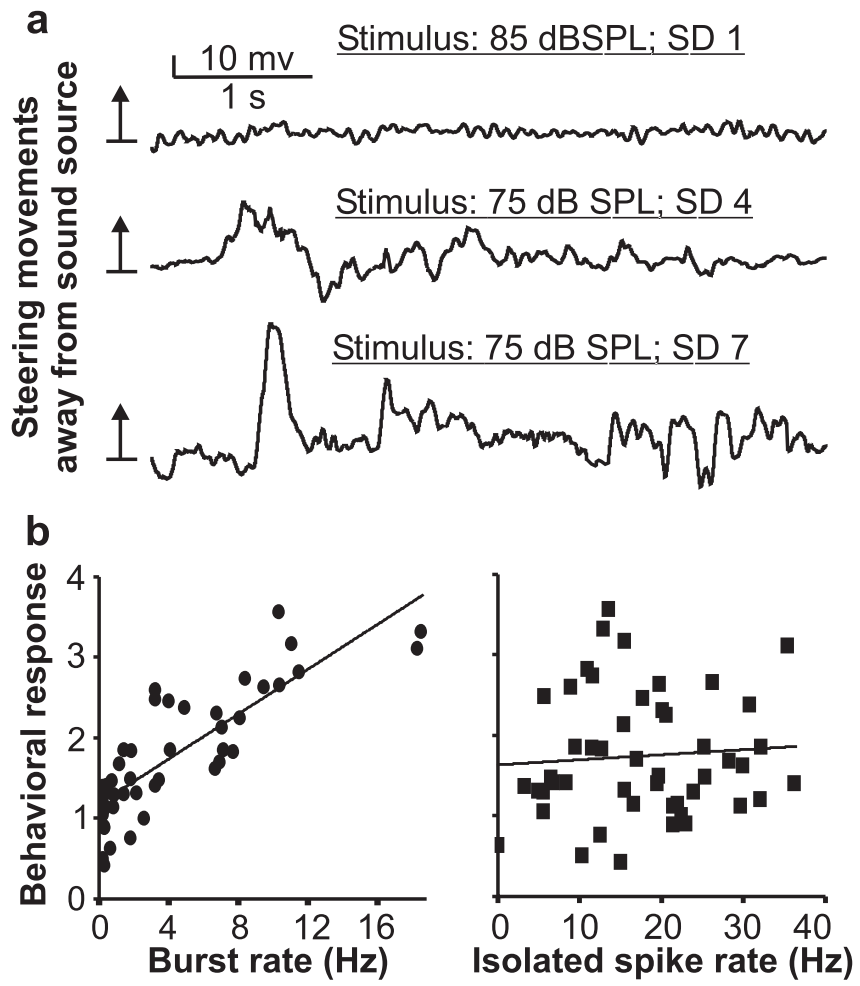

Figure 5. Behavioral responses vary with burst rate. $\boldsymbol{a}$, Excerpts of behavioral responses to stimuli that had the same modulation envelope but different mean intensity or modulation depth (indicated above each recording). The traces show abdominal movements (individual responses of the same cricket to different stimuli) beginning $6 \mathrm{~s}$ after sound onset (scale as in Fig. 4). In this animal, the mean response of the sound-ipsilateral AN2 in response to these stimuli was as follows, from top to bottom: 0.7, 7.2, and 10.4 bursts per second and 20.3, 19.1, and 26.4 isolated spikes per second. $\boldsymbol{b}$, Behavioral response, quantified as the SD of abdominal movements (millivolts of output of recording device), as a function of the isolated-spike rate (right) and burst rate (left) in the sound-ipsilateral AN2. Data points are from six experiments, in each of which stimuli with different mean sound levels (70-90 dB SPL) and/or modulation depths (envelope SD, 1-9 dB) were presented. Best-fit linear regression lines are shown. See supplemental Figures 1 and 2 (available at www.jneurosci.org as supplemental material).

differences in bursting of the two AN2s. These differences were apparent in both spike count and burst timing, both of which can provide cues for sound localization. It is not yet known, for responses to ultrasound, whether one or the other of these cues dominates in the determination of response direction. For positive phonotaxis to cricket songs, bilateral timing difference is less important than differences in response strength (Pollack, 2003; Hedwig and Poulet, 2005). Moreover, in our experiments, for more than three-quarters of the bursts in the sound-ipsilateral $\mathrm{AN} 2$, there was no burst in the contralateral neuron within a 10 $\mathrm{ms}$ window, thus obviating the need to compare binaural timing. The strong bilateral difference in bursting is attributable, in part, to contralateral inhibition. An identified interneuron, ON1, is excited by input from one ear and inhibits the AN2 that is excited by the other ear (Selverston et al., 1985). We showed previously that contralateral inhibition selectively suppresses high firing rates in AN2 (Marsat and Pollack, 2005) and thus should enhance binaural contrast preferentially for bursts.

Isolated spikes were common in responses by AN2 to the stimuli we used, yet they had little, if any, behavioral impact. AN2 also responds to cricket songs (Libersat et al., 1994) and contributes to behavioral responses to these stimuli (Schildberger and Hörner, 1988). Thus, isolated spikes may play a role in encoding these signals. The role of AN2 in bat evasion is known to be context dependent; it triggers avoidance steering only during flight (Nolen and Hoy, 1984). If isolated spikes play a role in responses to cricket song, then this would be another instance of the context-dependency of the behavioral impact of this neuron, the context in this case being the nature of the stimulus. For bat-evasion responses, however, isolated AN2 spikes may simply be "noise," which is filtered out by the fact that only bursts elicit strong behavioral responses. Noise filtering by bursts has been shown in other systems in which, because of synaptic summation or facilitation, bursts are particularly effective in driving their target cells (Boyan and Fullard, 1988; Lisman, 1997; Swadlow and Gusev, 2001). The same may be true of the interactions of AN2 with its target neurons.

\section{References}

Boyan GS, Fullard JH (1988) Information processing at a central synapse suggests a noise filter in the auditory pathway of the noctuid moth. J Comp Physiol A Neuroethol Sens Neural Behav Physiol 164:251-258.

Brodfuehrer PD, Hoy RR (1989) Integration of ultrasound and flight inputs on descending neurons in the cricket brain. J Exp Biol 145:157-171.

Chacron MJ, Longtin AE, Maler L (2004) To burst or not to burst? J Comput Neurosci 17:127-136.

Dean I, Harper NS, McAlpine D (2005) Neural population coding of sound level adapts to stimulus statistics. Nat Neurosci 8:1684-1689.

Eggermount JJ, Smith GM (1996) Burst-firing sharpens frequency-tuning in primary auditory cortex. NeuroReport 7:753-757.

Guido W, Lu SM, Vaughan JW, Godwin DW, Sherman SM (1995) Receiver operating characteristic (ROC) analysis of neurons in the cat's lateral geniculate nucleus during tonic and burst response mode. Vis Neurosci 12:723-741.

Hedwig B, Poulet JFA (2005) Mechanisms underlying phonotactic steering in the cricket Gryllus bimaculatus revealed with a fast trackball system. J Exp Biol 208:915:927.

Hoy RR (1992) The evolution of hearing in insects as an adaptation to predation from bats. In: The evolutionary biology of hearing (Webster DB, Fay RR, Popper AN, eds), pp 115-130. New York: Springer.

Krahe R, Gabbiani F (2004) Burst firing in sensory systems. Nat Rev Neurosci 5:13-23.

Kvale MN, Schreiner CE (2004) Short-term adaptation of auditory receptive fields to dynamic stimuli. J Neurophysiol 91:604-612.

Lesica NA, Stanley GB (2004) Encoding of natural scene movies by tonic and burst spikes in the lateral geniculate nucleus. J Neurosci 24:10731-10740.

Libersat F, Murray JA, Hoy RR (1994) Frequency as a releaser in the courtship song of two crickets, Gryllus bimaculatus (de Geer) and Teleogryllus oceanicus: a neuroethological analysis. J Comp Physiol A Neuroethol Sens Neural Behav Physiol 174:485-494.

Lisman JE (1997) Bursts as a unit of neural information: making unreliable synapse reliable. Trends Neurosci 20:38-43.

Marsat G, Pollack GS (2004) Differential temporal coding of rhythmically diverse acoustic signals by a single interneuron. J Neurophysiol 92:939-948.

Marsat G, Pollack GS (2005) Effect of the temporal pattern of contralateral inhibition on sound localization cues. J Neurosci 25:6137-6144.

Martinez-Conde S, Macknik SL, Hubel DH (2002) The function of bursts of spikes during visual fixation in the awake primate lateral geniculate nucleus and primary visual cortex. Proc Natl Acad Sci USA 99:13920-13925.

Metzner W, Koch C, Wessel R, Gabbiani F (1998) Feature extraction by burst-like spike patterns in multiple sensory maps. J Neurosci 18:2283-2300.

Moiseff A, Pollack GS, Hoy RR (1978) Steering responses of flying crickets to sound and ultrasound: mate attraction and predator avoidance. Proc Natl Acad Sci USA 75:4052-4056.

Nolen TG, Hoy RR (1984) Initiation of behavior by single neurons: the role of behavioral context. Science 226:992-994.

Nolen TG, Hoy RR (1986) Phonotaxis in flying crickets. I. Attraction to the calling song and avoidance of bat-like ultrasound are discrete behaviors. J Comp Physiol A Neuroethol Sens Neural Behav Physiol 159:423-439.

Oswald AM, Chacron MJ, Doiron B, Bastian J, Maler L (2004) Parallel processing of sensory input by bursts and isolated spikes. J Neurosci 24:4351-4362. 
Pollack GS (1998) Neural processing of acoustic signals. In: Comparative hearing: insects (Hoy RR, Popper AN, Fay RR, eds), pp 139-196. New York: Springer.

Pollack GS (2003) Sensory cues for sound localization in the cricket Teleogryllus oceanicus: interaural difference in response strength versus interaural latency difference. J Comp Physiol A Neuroethol Sens Neural Behav Physiol 189:143-151.

Pollack GS, Plourde N (1982) Directionality of acoustic orientation in flying crickets. J Comp Physiol A Neuroethol Sens Neural Behav Physiol 146:207-215.

Samson AH, Pollack GS (2002) Encoding of sound localization cues by an identified auditory interneuron: effects of stimulus temporal pattern. J Neurophysiol 88:2322-2328.

Schildberger K, Hörner M (1988) The function of auditory neurons in cricket phonotaxis. I. Influence of hyperpolarization of indentified neurons on sound localization. J Comp Physiol A Neuroethol Sens Neural Behav Physiol 163:621-631.
Selverston AI, Kleindienst HU, Huber F (1985) Synaptic connectivity between cricket auditory interneurons as studied by selective photoinactivation. J Neurosci 5:1283-1292.

Sherman SM (2001) Tonic and burst firing: dual modes of thalamocortical relay. Trends Neurosci 24:122-126.

Simmons JA (2005) Big brown bats and june beetles: multiple pursuit strategies in a seasonal acoustic predator-prey system. Acoust Res Lett Online $6: 238-242$

Smirnakis SM, Berry MJ, Warland DK, Bialek W, Meister M (1997) Adaptation of retinal processing to image contrast and spatial scale. Nature 386:69-73.

Swadlow HA, Gusev AG (2001) The impact of "bursting" thalamic impulses at a neocortical synapse. Nat Neurosci 4:402-408.

Yu AC, Margoliash D (1996) Temporal hierarchical control of singing in birds. Science 273:1871-1875.

Zar JH (1999) Biostatistical analysis, Ed 4. Upper Saddle River, NJ: PrenticeHall. 